\title{
NNLO jet cross sections by subtraction
}

\author{
G. Somogyi ${ }^{\mathrm{a}}$, P. Bolzoni ${ }^{\mathrm{a}}$, Z. Trócsányi ${ }^{\mathrm{b}}$

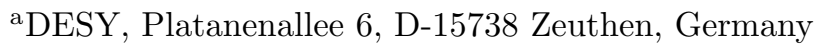 \\ ${ }^{\mathrm{b}}$ CERN PH-TH, on leave from University of Debrecen and Institute of Nuclear Research of HAS, \\ H-4001 P.O.Box 51, Hungary
}

We report on the computation of a class of integrals that appear when integrating the so-called iterated singlyunresolved approximate cross section of the NNLO subtraction scheme of Refs. [1-4] over the factorised phase space of unresolved partons. The integrated approximate cross section itself can be written as the product of an insertion operator (in colour space) times the Born cross section. We give selected results for the insertion operator for processes with two and three hard partons in the final state.

\section{Introduction}

High energy particle collisions frequently lead to final states with hadronic jets. Jet observables can be ideal for precision studies, since their large production cross sections allow them to be measured with high statistical accuracy. Examples include: the determination of the strong coupling, $\alpha_{\mathrm{s}}$, from jet rates and event shapes in electronpositron annihilation; the measurement of gluon parton distribution functions (and also $\alpha_{\mathrm{s}}$ ) in deep inelastic lepton-hadron scattering into two plus one jets; the determination of parton distributions in hadron-hadron scattering from single jet inclusive production and vector boson plus jet production. The relevant observables are often measured with experimental precision of a few per cent or better, thus theoretical predictions with the same level of accuracy are necessary. This usually requires the computation of next-to-nextto-leading order (NNLO) corrections in perturbative QCD.

One of the main bottlenecks of the straightforward application of QCD perturbation theory at NNLO is the following. Due to the presence of infrared (IR) singularities, the finite higher-order corrections are sums of pieces which are separately divergent in $d=4$ spacetime dimensions. These IR singularities must be regularised and cancelled before any numerical computation may be performed. The most common approach for handling IR singularities at next-to-leading order (NLO) accuracy is the subtraction method. After regularising all contributions by dimensional regularisation in $d=4-2 \epsilon$ dimensions, one builds subtraction terms that simultaneously cancel both the kinematical singularities in realemission phase space integrals and the explicit $\epsilon$-poles in one-loop virtual corrections [5]. Since the IR singularity structure of QCD amplitudes is universal, such subtraction terms can be defined in a general, i.e. process and observable independent fashion.

In recent years, severe efforts have been made to extend the subtraction method to NNLO accuracy [1-4,6-12], and this has proved to be a challenging problem. In broad terms, any proposed subtraction scheme must address two quite distinct difficulties. First, one must define subtraction terms that properly regularise the realemission phase space integrals. In a rigorous mathematical sense, the cancellation of kinematical singularities must be local, i.e. the subtraction terms must have the same pointwise singular behaviour in $d$ dimensions, as the real-emission pieces. Second, one must combine the integrated form of these counterterms with the virtual contributions, cancelling the IR divergences of the loop matrix elements. Again, from the point of view of mathematical rigour, this cancellation must be local, i.e. it must happen pointwise in phase space. Practically, the full locality of the 
subtraction scheme is also important to ensure good numerical efficiency of the algorithm. Finally, the construction should be universal (process and observable independent), otherwise tedious adaptation of the algorithm to every specific problem becomes necessary.

The construction of a general subtraction scheme with fully local counterterms requires a lot of careful analytical calculations. In order to spare some work, various approaches have been proposed to NNLO calculations sacrificing either full locality or generality for easier analytical (but more cumbersome numerical) treatment. For example, in the antenna subtraction scheme [9-12] azimuthal correlations in gluon splitting are not reproduced (and the cancellation of $\epsilon$-poles in the real-virtual contributions is also nonlocal). As a consequence, actual numerical computation of total rates $[13-16]$ and event shapes [17-21] in $e^{+} e^{-} \rightarrow 3$ jet production in the antenna scheme requires the use of an auxiliary phase space slicing. Another option is to develop dedicated subtraction schemes that are applicable only to some specific processes, such as the production of colourless final states in hadron-hadron collisions [22-24]. Then, one may even propose to dispense with the subtraction method altogether, and adopt a strategy such as sector decomposition (see e.g. [25] and references therein).

Nevertheless, it is possible to address all subtleties, and define completely local counterterms for real radiation, as done in full detail in Refs. [1-4] for the case of colourless initial states. (Work towards an extension to hadron-initiated processes is presented in Ref. [26].) Then, to make this subtraction scheme an effective tool, one must compute the integrals of all subtraction terms over the phase spaces of unresolved emissions. In this contribution, we briefly review the methods used to compute the integrated subtraction terms, and report on the evaluation of the integrated iterated singly-unresolved cross section, the term labelled $\mathrm{A}_{12}$, which appears in Eq. (5). However, we begin by recalling the essentials of the subtraction scheme of Refs. [1-4].

\section{Subtraction at NNLO}

Consider the NNLO correction to a generic $m$ jet observable,

$$
\begin{aligned}
\sigma^{\mathrm{NNLO}} & =\int_{m+2} \mathrm{~d} \sigma_{m+2}^{\mathrm{RR}} J_{m+2}+\int_{m+1} \mathrm{~d} \sigma_{m+1}^{\mathrm{RV}} J_{m+1} \\
& +\int_{m} \mathrm{~d} \sigma_{m}^{\mathrm{VV}} J_{m} .
\end{aligned}
$$

The three contributions on the right hand side are separately divergent in $d=4$ dimensions, but their sum is finite for IR safe observables. To obtain the finite NNLO correction, we first continue analytically all integrals to $d=4-2 \epsilon$ dimensions and then rewrite Eq. (1) as

$$
\begin{aligned}
\sigma^{\mathrm{NNLO}} & =\int_{m+2} \mathrm{~d} \sigma_{m+2}^{\mathrm{NNLO}}+\int_{m+1} \mathrm{~d} \sigma_{m+1}^{\mathrm{NNLO}} \\
& +\int_{m} \mathrm{~d} \sigma_{m}^{\mathrm{NNLO}}
\end{aligned}
$$

that is a sum of three finite integrals where the integrands,

$$
\begin{gathered}
\mathrm{d} \sigma_{m+2}^{\mathrm{NNLO}}=\left\{\mathrm{d} \sigma_{m+2}^{\mathrm{RR}} J_{m+2}-\mathrm{d} \sigma_{m+2}^{\mathrm{RR}, \mathrm{A}_{2}} J_{m}\right. \\
\left.-\left[\mathrm{d} \sigma_{m+2}^{\mathrm{RR}, \mathrm{A}_{1}} J_{m+1}-\mathrm{d} \sigma_{m+2}^{\mathrm{RR}, \mathrm{A}_{12}} J_{m}\right]\right\}_{\epsilon=0}, \\
\mathrm{~d} \sigma_{m+1}^{\mathrm{NNLO}}=\left\{\left[\mathrm{d} \sigma_{m+1}^{\mathrm{RV}}+\int_{1} \mathrm{~d} \sigma_{m+2}^{\mathrm{RR}, \mathrm{A}_{1}}\right] J_{m+1}\right. \\
\left.-\left[\mathrm{d} \sigma_{m+1}^{\mathrm{RV}, \mathrm{A}_{1}}+\left(\int_{1} \mathrm{~d} \sigma_{m+2}^{\mathrm{RR}, \mathrm{A}_{1}}\right)^{\mathrm{A}_{1}}\right] J_{m}\right\}_{\epsilon=0},
\end{gathered}
$$

and

$$
\begin{aligned}
& \mathrm{d} \sigma_{m}^{\mathrm{NNLO}}=\left\{\mathrm{d} \sigma_{m}^{\mathrm{VV}}+\int_{2}\left[\mathrm{~d} \sigma_{m+2}^{\mathrm{RR}, \mathrm{A}_{2}}-\mathrm{d} \sigma_{m+2}^{\mathrm{RR}, \mathrm{A}_{12}}\right]\right. \\
& \left.+\int_{1}\left[\mathrm{~d} \sigma_{m+1}^{\mathrm{RV}, \mathrm{A}_{1}}+\left(\int_{1} \mathrm{~d} \sigma_{m+2}^{\mathrm{RR}, \mathrm{A}_{1}}\right)^{\mathrm{A}_{1}}\right]\right\}_{\epsilon=0} J_{m},
\end{aligned}
$$

are integrable in four dimensions by construction. The approximate cross sections $\mathrm{d} \sigma_{m+2}^{\mathrm{RR}, \mathrm{A}_{2}}$ and $\mathrm{d} \sigma_{m+2}^{\mathrm{RR}, \mathrm{A}_{1}}$ regularise the doubly- and singlyunresolved limits of the real-emission piece, $\mathrm{d} \sigma_{m+2}^{\mathrm{RR}}$ respectively. The double subtraction due to the overlap of these two terms is compensated by $\mathrm{d} \sigma_{m+2}^{\mathrm{RR}, \mathrm{A}_{12}}$. These terms are given explicitly in 
Ref. [3]. Finally, $\mathrm{d} \sigma_{m+1}^{\mathrm{RV}, \mathrm{A}_{1}}$ and $\left(\int_{1} \mathrm{~d} \sigma_{m+2}^{\mathrm{RR}, \mathrm{A}_{1}}\right)^{\mathrm{A}_{1}}$ regularise the singly-unresolved limits of $\mathrm{d} \sigma_{m+1}^{\mathrm{RV}}$ and $\int_{1} \mathrm{~d} \sigma_{m+2}^{\mathrm{RR}, \mathrm{A}_{1}}$ respectively. They are given explicitly in Ref. [4].

The construction of each approximate cross section in Eqs. (3)-(5) is based on the known and universal IR limits of tree level and one-loop squared matrix elements, and proceeds in two steps. First, the IR factorisation formulae are written in such a way that their complicated overlap structure can be disentangled ("matching of limits") $[1,27]$. Second, we define "extensions" of the formulae, so that they are unambiguously defined away from the strict IR limits [2-4]. These extensions are defined by the use of various momentum mappings that map a set of $m+1$ or $m+2$ momenta into a set of $m$ momenta,

$\{p\}_{m+1} \longrightarrow\{\tilde{p}\}_{m}$ and $\{p\}_{m+2} \longrightarrow\{\tilde{p}\}_{m}$,

such that (i) the delicate structure of cancellations among the matched limit formulae in various limits is respected (ii) exact momentum conservation is implemented, and (iii) the original $m+1$ or $m+2$ particle phase space factorises exactly into the product of an $m$ particle phase space and a one- or two-particle phase space measure,

$\mathrm{d} \phi_{m+1}\left(\{p\}_{m+1} ; Q\right)=\mathrm{d} \phi_{m}\left(\{\tilde{p}\}_{m} ; Q\right)\left[\mathrm{d} p_{1, m}\right]$

and

$\mathrm{d} \phi_{m+2}\left(\{p\}_{m+2} ; Q\right)=\mathrm{d} \phi_{m}\left(\{\tilde{p}\}_{m} ; Q\right)\left[\mathrm{d} p_{2, m}\right]$.

To finish the definition of the scheme, one must compute once and for all the one- and two-particle integrals, denoted formally as $\int_{1}$ and $\int_{2}$, appearing in Eqs. (4) and (5). We discuss this next.

\section{Integrating the counterterms}

The actual computation of the integrated counterterms leads to a large number of multidimensional integrals. The ultimate goal is to find the analytical form of the coefficients of a Laurent-expansion (in $\epsilon$ ) of these integrals, which turns out to be a rather a tedious job. In order to compute these coefficients as efficiently as possible, we have explored several methods.
First, it is possible to extend the method of integration-by-parts identities and solving of differential equations, developed for computing multi-loop Feynman integrals [28, 29], to the relevant phase space integrations [30]. This method yields $\epsilon$-expansions with fully analytical coefficients, with the final results being expressed in terms of two-dimensional harmonic polylogarithms (after a suitable basis extension, see Ref. [30] for details). This approach was used successfully to compute a class of singly-unresolved integrals [30].

Second, the phase space integrals that arise can be computed via the method of Mellin-Barnes (MB) representations [31-33]. Here we obtain the $\epsilon$-expansion coefficients in terms of complex contour integrals over $\Gamma$-functions. Performing these integrals by the use of the residue theorem, a representation in terms of harmonic sums is obtained. In many cases, the sums can be evaluated in a closed form, yielding an analytical result. In some instances however, we find multi-dimensional MB integrals that are very difficult to compute fully analytically. Nevertheless, in these situations a direct numerical evaluation of the appropriate MB representations provides a fast and reliable way to obtain final results with small numerical uncertainties. We stress that for phenomenological applications, this is all that is required, since the relative numerical uncertainty associated with phase space integrations is generally much larger than that of the integrated counterterms. We have used the MB method to compute all singly-unresolved integrals [34], and very recently to evaluate all two-particle integrals appearing in $\int_{2} \mathrm{~d} \sigma_{m+2}^{\mathrm{RR}, \mathrm{A}_{12}}$ as well [35] (see below).

Finally, the method of iterated sector decomposition [25] can also be used to calculate the integrals we encounter [36]. Sector decomposition produces a representation of the $\epsilon$-expansion where the coefficients are given in terms of (mostly quite cumbersome) finite integrals over the unit hypercube. The analytical evaluation of these integrals is not feasible except for the simplest cases, and we find that in most instances, the MB method provides an integral representation for the expansion coefficients which is better 
suited for direct numerical integration. Nevertheless, this method is simple to implement and we employed it to numerically cross check all our final results.

\section{Results}

In previous publications, all one-particle integrals, denoted formally by $\int_{1}$ in Eqs. (4) and (5) have been evaluated with the methods just discussed $[30,34,36]$. Here we report on the computation of the $\int_{2} \mathrm{~d} \sigma_{m+2}^{\mathrm{RR}, \mathrm{A}_{12}}$ two-particle integrated counterterm [35]. To begin, we recall that the iterated singly-unresolved subtraction term, $\mathrm{d} \sigma_{m+2}^{\mathrm{RR}, \mathrm{A}_{12}}$ can be written symbolically as [3]

$\mathrm{d} \sigma_{m+2}^{\mathrm{RR}, \mathrm{A}_{12}}=\mathrm{d} \phi_{m}\left[\mathrm{~d} p_{1, m}\right]\left[\mathrm{d} p_{1, m+1}\right] \mathcal{A}_{12}\left|\mathcal{M}_{m+2}^{(0)}\right|^{2}$,

where $\mathcal{A}_{12}\left|\mathcal{M}_{m+2}^{(0)}\right|^{2}$ further evaluates to a sum of several terms, as spelt out in detail in Ref. [3]. Each of these terms is essentially a product of kinematical factors times an $m$-parton factorised matrix element, the later being independent of the variables in the factorised phase space measure $\left[\mathrm{d} p_{1, m}\right]\left[\mathrm{d} p_{1, m+1}\right]$. Thus, it is possible to evaluate the integrals of the kinematical factors over the factorised phase spaces, without reference to the specific process or observable. The final result, after summation over unobserved flavours, can be written in the form of an insertion operator (in colour space) times the Born cross section

$\int_{2} \mathrm{~d} \sigma_{m+2}^{\mathrm{RR}, \mathrm{A}_{12}}=\mathrm{d} \sigma_{m}^{\mathrm{B}} \otimes \boldsymbol{I}_{12}^{(0)}\left(\{p\}_{m} ; \epsilon\right)$.

Here the insertion operator has three terms according to the possible colour structures

$$
\begin{aligned}
\boldsymbol{I}_{12}^{(0)} & =\left[\frac{\alpha_{\mathrm{s}}}{2 \pi} S_{\epsilon}\left(\frac{\mu^{2}}{Q^{2}}\right)^{\epsilon}\right]^{2} \\
& \times\left\{\sum_{i}\left[\mathrm{C}_{12, f_{i}}^{(0)} C_{f_{i}}+\sum_{k \neq i} \mathrm{C}_{12, f_{i} f_{k}}^{(0)} C_{f_{k}}\right] C_{f_{i}}\right. \\
& +\sum_{j, l \neq j}\left[\mathrm{~S}_{12}^{(0),(j, l)} C_{\mathrm{A}}+\sum_{i} \mathrm{CS}_{12, f_{i}}^{(0),(j, l)} C_{f_{i}}\right] \boldsymbol{T}_{j} \boldsymbol{T}_{l}
\end{aligned}
$$

$$
\left.+\sum_{i, k \neq i} \sum_{j, l \neq j} \mathrm{~S}_{12}^{(0),(i, k)(j, l)}\left\{\boldsymbol{T}_{i} \boldsymbol{T}_{k}, \boldsymbol{T}_{j} \boldsymbol{T}_{l}\right\}\right\},
$$

with $f_{i}$ denoting flavours, $C_{q}=C_{\mathrm{F}} \equiv \boldsymbol{T}_{q}^{2}, C_{g}=$ $C_{\mathrm{A}} \equiv \boldsymbol{T}_{g}^{2}$, and $S_{\epsilon}=\frac{(4 \pi)^{\epsilon}}{\Gamma(1-\epsilon)}$. In Eq. (11), the dependence of the functions $\mathrm{C}_{12, f_{i}}^{(0)}$, etc. on the kinematics is suppressed for the sake of simplicity. These functions in turn are given as the following combinations of flavour summed integrated counterterms

$$
\begin{aligned}
& \mathrm{C}_{12, f_{i}}^{(0)}= \\
& =\left(\mathrm{C}_{k t} \mathrm{C}_{k t r}^{(0)}\right)_{f_{i}}-\left(\mathrm{C}_{k t} \mathrm{C}_{k t r} \mathrm{CS}_{k t ; r}^{(0)}\right)_{f_{i}} \\
& -\left(\mathrm{C}_{k t} \mathrm{C}_{r k t} \mathrm{~S}_{k t}^{(0)}\right)_{f_{i}}+\left(\mathrm{S}_{t} \mathrm{C}_{i r t}^{(0)}\right)_{f_{i}} \\
& -\left(\mathrm{S}_{t} \mathrm{C}_{i r t} \mathrm{CS}_{i r ; t}^{(0)}\right)_{f_{i}}-\left(\mathrm{S}_{t} \mathrm{C}_{i r t} \mathrm{~S}_{r t}^{(0)}\right)_{f_{i}} \\
& +\left(\mathrm{S}_{t} \mathrm{C}_{i r t} \mathrm{CS}_{i r ; t} \mathrm{~S}_{r t}^{(0)}\right)_{f_{i}}-\left(\mathrm{C}_{k t} \mathrm{~S}_{t} \mathrm{C}_{k r t}^{(0)}\right)_{f_{i}} \\
& +\left(\mathrm{C}_{k t} \mathrm{~S}_{t} \mathrm{C}_{k r t} \mathrm{~S}_{r t}^{(0)}\right)_{f_{i}}, \\
& \mathrm{C}_{12, f_{i} f_{k}}^{(0)}={ }_{(13)} \\
& \quad=\left(\mathrm{C}_{k t} \mathrm{C}_{i r ; k t}^{(0)}\right)_{f_{i} f_{k}}-\left(\mathrm{C}_{k t} \mathrm{C}_{i r ; k t} \mathrm{CS}_{k t ; r}^{(0)}\right)_{f_{i} f_{k}} \\
& \quad-\left(\mathrm{C}_{k t} \mathrm{~S}_{t} \mathrm{CS}_{i r ; t}^{(0)}\right)_{f_{i} f_{k}}+\left(\mathrm{C}_{k t} \mathrm{~S}_{t} \mathrm{CS}_{i r ; t} \mathrm{~S}_{r t}^{(0)}\right)_{f_{i} f_{k}},
\end{aligned}
$$

$$
\begin{aligned}
& \mathrm{S}_{12}^{(0),(j, l)}= \\
& =\left(\mathrm{C}_{k t} \mathrm{~S}_{k t}^{(0)}\right)^{(j, l)}+\left(\mathrm{S}_{t} \mathrm{~S}_{r t}^{(0)}\right)^{(j, l)} \\
& \quad-\left(\mathrm{C}_{k t} \mathrm{~S}_{t} \mathrm{~S}_{k t}^{(0)}\right)^{(j, l)} \\
& \mathrm{CS}_{12, f_{i}}^{(0),(j, l)}= \\
& \quad=\left(\mathrm{C}_{k t} \mathrm{CS}_{k t ; r}^{(0)}\right)_{f_{i}}^{(j, l)}+\left(\mathrm{S}_{t} \mathrm{CS}_{i r ; t}^{(0)}\right)_{f_{i}}^{(j, l)} \\
& \quad-\left(\mathrm{S}_{t} \mathrm{CS}_{i r ; t} \mathrm{~S}_{r t}^{(0)}\right)_{f_{i}}^{(j, l)}-\left(\mathrm{C}_{k t} \mathrm{~S}_{t} \mathrm{~S}_{r t}^{(0)}\right)_{f_{i}}^{(j, l)}
\end{aligned}
$$


$\mathrm{S}_{12}^{(0),(i, k)(j, l)}=\left(\mathrm{S}_{t} \mathrm{~S}_{r t}^{(0)}\right)^{(i, k)(j, l)}$.

On the right hand sides of these equations, the flavour summed integrated counterterms themselves are sums of various integrated subtraction terms. Their precise definitions are somewhat long, and will not be reported here.

Instead, we present results for the insertion operator in Eq. (11) for processes with two and three partons in the final state. Consider first the process $e^{+} e^{-} \rightarrow 2$ jets. The corresponding Born matrix element is $\left|\mathcal{M}_{2}^{(0)}\left(1_{q}, 2_{\bar{q}}\right)\right|^{2}$, i.e. the quark carries the label 1 and antiquark label 2 . The colour algebra is trivial [5], and all properly scaled kinematical invariants are equal to one, i.e. $\boldsymbol{I}_{12}^{(0)}\left(\{p\}_{2}\right)$ does not depend on the kinematics. Introducing the notation [37] $x=C_{\mathrm{A}} / C_{\mathrm{F}}, y=T_{\mathrm{R}} / C_{\mathrm{F}}$, we find

$$
\begin{aligned}
\boldsymbol{I}_{12}^{(0)}( & \left.\{p\}_{2}\right)=\left[\frac{\alpha_{\mathrm{s}}}{2 \pi} S_{\epsilon}\left(\frac{\mu^{2}}{Q^{2}}\right)^{\epsilon}\right]^{2} C_{\mathrm{F}}^{2}\left\{\frac{6-2 x}{\epsilon^{4}}\right. \\
& +\left[12+\frac{7 x}{2}+y n_{\mathrm{f}}-(4-4 x) \Sigma\left(y_{0}, D_{0}^{\prime}-1\right)\right. \\
& \left.\left.-(4-6 x) \Sigma\left(y_{0}, D_{0}^{\prime}\right)\right] \frac{1}{\epsilon^{3}}+\mathrm{O}\left(\epsilon^{-2}\right)\right\}
\end{aligned}
$$

where $y_{0} \in(0,1]$ and $D_{0}^{\prime} \geq 2$ and integer are fixed parameters that enter the precise definition of the subtraction terms. Finally, the function $\Sigma(z, N)$ is defined as follows

$\Sigma(z, N)=\ln z-\sum_{k=1}^{N} \frac{1-(1-z)^{k}}{k}$.

Next, consider $e^{+} e^{-} \rightarrow 3$ jet production. The Born matrix element is $\left|\mathcal{M}_{3}^{(0)}\left(1_{q}, 2_{\bar{q}}, 3_{g}\right)\right|^{2}$, i.e. the quark carries label 1 , the antiquark label 2 and the gluon carries label 3. The colour algebra is still trivial [5], but $\boldsymbol{I}_{12}^{(0)}\left(\{p\}_{3}\right)$ now carries genuine kinematical dependence. We find

$$
\begin{aligned}
\boldsymbol{I}_{12}^{(0)} & \left(\{p\}_{3}\right)=\left[\frac{\alpha_{\mathrm{s}}}{2 \pi} S_{\epsilon}\left(\frac{\mu^{2}}{Q^{2}}\right)^{\epsilon}\right]^{2} C_{\mathrm{F}}^{2}\left\{\frac{6+2 x+x^{2}}{\epsilon^{4}}\right. \\
& +\left[12+\frac{101 x}{6}+\frac{67 x^{2}}{12}-\frac{13 y}{3} n_{\mathrm{f}}-\frac{3 x y}{2} n_{\mathrm{f}}\right. \\
& -\left(4 x+\frac{5 x^{2}}{2}\right)\left(\ln y_{13}+\ln y_{23}\right)
\end{aligned}
$$

$$
\begin{aligned}
& -\left(8+x-\frac{5 x^{2}}{2}\right) \ln y_{12} \\
& -(4-4 x) \Sigma\left(y_{0}, D_{0}^{\prime}-1\right) \\
& \left.\left.-\left(4-6 x-x^{2}\right) \Sigma\left(y_{0}, D_{0}^{\prime}\right)\right] \frac{1}{\epsilon^{3}}+\mathrm{O}\left(\epsilon^{-2}\right)\right\} .
\end{aligned}
$$

In this equation $y_{i k}=2 p_{i} \cdot p_{k} / Q^{2}$, with $Q^{\mu}$ being the total incoming momentum. Higher order expansion coefficients (in $\epsilon$ ) in Eq. (19) are already quite cumbersome, and will be given elsewhere in the form of a computer program [35].

\section{Conclusions}

In this contribution, we have reported the computation of the integrated iterated singly-unresolved approximate cross section, $\int_{2} \mathrm{~d} \sigma_{m+2}^{\mathrm{RR}, \mathrm{A}_{12}}$, that appears in the NNLO subtraction scheme of Refs. [2-4]. The phase space integrals appearing in the computation can be evaluated once and for all, and their knowledge (as Laurent expansions in $\epsilon$, up to and including $\mathrm{O}\left(\epsilon^{0}\right)$ terms) is necessary to make the subtraction scheme an effective tool. All phase space integrals were evaluated with two separate methods. First, we used the method of Mellin-Barnes representations with harmonic summation to obtain analytical results where feasible. In some cases, obtaining complete analytical answers is very difficult. In these situations, we integrated the MB representations of the expansion coefficients directly, and this provides a fast and reliable way to obtain final results with small numerical uncertainties. Second, all integrals were computed with the method of sector decomposition as well, providing useful numerical checks.

With the evaluation of $\int_{2} \mathrm{~d} \sigma_{m+2}^{\mathrm{RR}, \mathrm{A}_{12}}$, the last task in finishing the definition of the subtraction scheme is the computation of the integrated doubly-unresolved approximate cross section, i.e. the term labelled $\mathrm{A}_{2}$ in Eq. (5). The analytical structure and complexity of the integrals that appear in this final piece are essentially the same as the integrals considered here. Therefore, the techniques outlined above will also be applicable to the computation of this remaining contribution.

This work was supported in part by the Deutsche Forschungsgemeinschaft in SFB/TR 9, 
the Helmholtz Gemeinschaft under contract VHNG-105, the Hungarian Scientific Research Fund grand OTKA K-60432.

\section{REFERENCES}

1. G. Somogyi, Z. Trócsányi, and V. Del Duca, JHEP 06, 024 (2005), arXiv:hep-ph/0502226.

2. G. Somogyi and Z. Trócsányi, (2006), arXiv:hep-ph/0609041.

3. G. Somogyi, Z. Trócsányi, and V. Del Duca, JHEP 01, 070 (2007), arXiv:hep-ph/0609042.

4. G. Somogyi and Z. Trócsányi, JHEP 01, 052 (2007), arXiv:hep-ph/0609043.

5. S. Catani and M. H. Seymour, Nucl. Phys. B485, 291 (1997), arXiv:hep-ph/9605323.

6. S. Weinzierl, JHEP 03, 062 (2003), arXiv:hep-ph/0302180.

7. S. Weinzierl, JHEP 07, 052 (2003), arXiv:hep-ph/0306248.

8. S. Frixione and M. Grazzini, JHEP 06, 010 (2005), arXiv:hep-ph/0411399.

9. A. Gehrmann-De Ridder, T. Gehrmann, and E. W. N. Glover, JHEP 09, 056 (2005), arXiv:hep-ph/0505111.

10. A. Daleo, T. Gehrmann, and D. Maitre, JHEP 04, 016 (2007), arXiv:hep-ph/0612257.

11. A. Daleo, A. Gehrmann-De Ridder, T. Gehrmann, and G. Luisoni, JHEP 01, 118 (2010), arXiv:0912.0374.

12. E. W. N. Glover and J. Pires, (2010), arXiv:1003.2824.

13. A. Gehrmann-De Ridder, T. Gehrmann, E. W. N. Glover, and G. Heinrich, JHEP 11, 058 (2007), arXiv:0710.0346.

14. A. Gehrmann-De Ridder, T. Gehrmann, E. W. N. Glover, and G. Heinrich, Phys. Rev. Lett. 100, 172001 (2008), arXiv:0802.0813.

15. S. Weinzierl, Phys. Rev. Lett. 101, 162001 (2008), arXiv:0807.3241.

16. S. Weinzierl, JHEP 07, 009 (2009), arXiv:0904.1145.

17. A. Gehrmann-De Ridder, T. Gehrmann, E. W. N. Glover, and G. Heinrich, Phys. Rev. Lett. 99, 132002 (2007), arXiv:0707.1285.

18. A. Gehrmann-De Ridder, T. Gehrmann, E. W. N. Glover, and G. Heinrich, JHEP 12, 094 (2007), arXiv:0711.4711.
19. A. Gehrmann-De Ridder, T. Gehrmann, E. W. N. Glover, and G. Heinrich, JHEP 05, 106 (2009), arXiv:0903.4658.

20. S. Weinzierl, JHEP 06, 041 (2009), arXiv:0904.1077.

21. S. Weinzierl, Phys. Rev. D80, 094018 (2009), arXiv:0909.5056.

22. S. Catani and M. Grazzini, Phys. Rev. Lett. 98, 222002 (2007), arXiv:hep-ph/0703012.

23. S. Catani, L. Cieri, G. Ferrera, D. de Florian, and M. Grazzini, Phys. Rev. Lett. 103, 082001 (2009), arXiv:0903.2120.

24. S. Catani, G. Ferrera, and M. Grazzini, JHEP 05, 006 (2010), arXiv:1002.3115.

25. G. Heinrich, Int. J. Mod. Phys. A23, 1457 (2008), arXiv:0803.4177.

26. G. Somogyi, JHEP 05, 016 (2009), arXiv:0903.1218.

27. Z. Nagy, G. Somogyi, and Z. Trócsányi, (2007), arXiv:hep-ph/0702273.

28. A. V. Kotikov, Phys. Lett. B267, 123 (1991).

29. E. Remiddi, Nuovo Cim. A110, 1435 (1997), arXiv:hep-th/9711188.

30. U. Aglietti, V. Del Duca, C. Duhr, G. Somogyi, and Z. Trócsányi, JHEP 09, 107 (2008), arXiv:0807.0514.

31. V. A. Smirnov, Phys. Lett. B460, 397 (1999), arXiv:hep-ph/9905323.

32. J. B. Tausk, Phys. Lett. B469, 225 (1999), arXiv:hep-ph/9909506.

33. V. A. Smirnov, Springer Tracts Mod. Phys. 211, 1 (2004).

34. P. Bolzoni, S.-O. Moch, G. Somogyi, and Z. Trócsányi, JHEP 08, 079 (2009), arXiv:0905.4390.

35. P. Bolzoni, G. Somogyi, and Z. Trócsányi, (2010), in preparation.

36. G. Somogyi and Z. Trócsányi, JHEP 08, 042 (2008), arXiv:0807.0509.

37. Z. Nagy and Z. Trócsányi, Phys. Rev. D59, 014020 (1999), arXiv:hep-ph/9806317. 